\title{
Estimation of Soil Electrical Conductivity using Dual - Polarized SAR Sentinel -1 Imagery
}

\author{
Tanvi Agarwal \\ Madhav Institute of Technology \& \\ Science \\ Race Course Road, Gole Ka \\ Mandir, Gwalior - 474005, Madhya \\ Pradesh, India
}

\author{
Rajni Ranjan Singh Makwana \\ Madhav Institute of Technology \& \\ Science \\ Race Course Road, Gole Ka \\ Mandir, Gwalior - 474005, Madhya \\ Pradesh, India
}

\author{
Laxmi Shrivastava \\ Madhav Institute of Technology \& \\ Science \\ Race Course Road, Gole Ka \\ Mandir, Gwalior - 474005, Madhya \\ Pradesh, India
}

\begin{abstract}
Soil to mankind is a basic natural resource. Soil is a blend of solid, liquid and gaseous substances, shapes the top most layer of the Earth's crust. The saline soil are the 'salt affected soils' generally found in arid and semi - arid regions. These soils are generally found in 'low precipitation area' where precipitation and evaporation ratio is less than 10.75[5]. This paper manages soil electrical conductivity estimation utilizing Sentinel -1 SAR imagery.. The support vector regression (SVR) technique, with RBF kernel function, was utilized to relate illustrative factors to ground estimated saltiness. We additionally applied K-Fold method for upgrading the model..
\end{abstract}

\section{General Terms}

Regression, Algorithm

\section{Keywords}

SAR imagery, Support Vector Regression, microwave, soil, electrical conductivity

\section{INTRODUCTION}

Salinization of soil is one of the most significant ecological issues in dry and semi-dry zones.[4]. Microwave remote detecting on regular earth material, for example, soil and water has a nearby reliance on their electrical parameters[2]. Nonetheless, few examinations have explored the capability of SAR imagery for saltiness checking[6]. Radar backscattering is for the most part impacted by two primary variables : (1) sensor parameters to be specific wavelength, polarization, incidence angle, and (2) target parameters, for example, surface roughness, slope orientation, and dielectric properties of an objective[7]. Soil complex dielectric permittivity range relies mostly upon soil volumetric water substance and saltiness, however other soil properties, for example, temperature, density and clay content can likewise altogether affect its dielectric properties[3]. This paper aims to evaluate the chance of utilizing radar imagery to recognize salinization

\section{SUPPORT VECTOR REGRESSION}

This algorithm is utilized to forecast continual parameter[1]. It tries to fit the best line within a pre-defined or threshold error value. As it can be seen from figure 1 that this algorithm takes 28 samples (x1, x2,.., x28 etc.) as input and $\mathrm{K}(\mathrm{xi}, \mathrm{xj})$ represents the kernel function, where $x i$, $x j$ are sample data and $\mathrm{Y}$ represents the output i.e. EC.

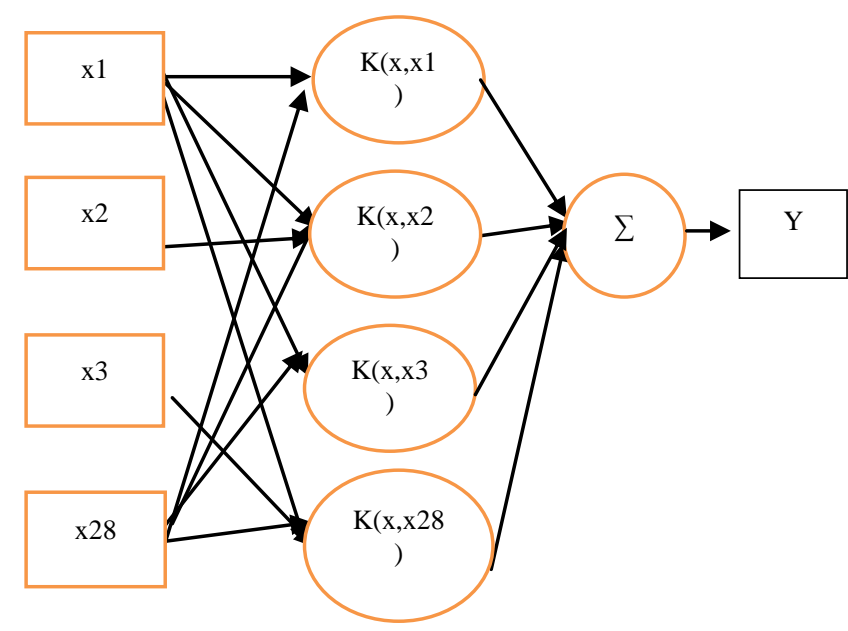

Figure 1. Structure of SVR algorithm

\section{K - FOLD CROSS VALIDATION}

Cross validation is a resampling strategy to assess artificial intelligence models on a finite data sets[10]. The technique has a single parameter $\mathrm{K}$ that alludes to the number of groups that a given data sets is to be divided into.

\section{MATERIALS AND METHODS}

\subsection{Study Area and Datasets}

Kuh Sefid is a town situated in the central district of Qom country as shown in figure 2. This zone has hot and dry atmosphere with yearly rainfall of $115.5 \mathrm{~mm}$, this region is influenced by serious saltiness risk predominantly because of region to the salt lake Qom.

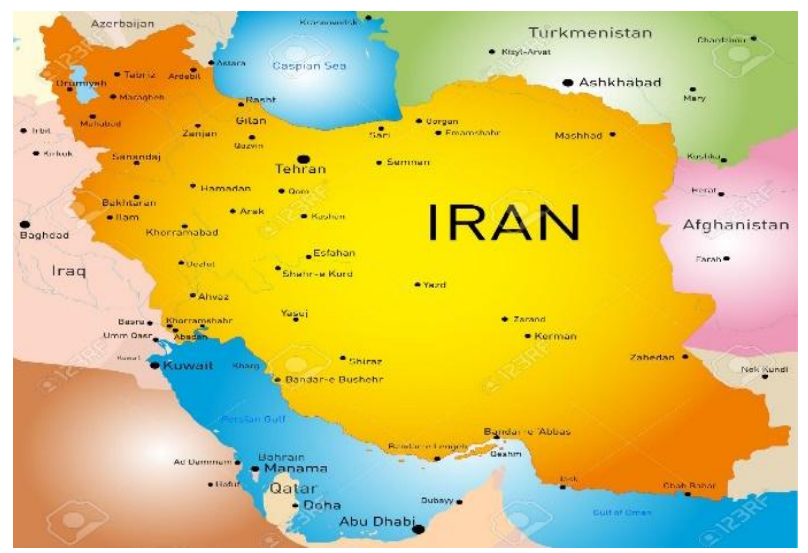

Figure 2. Location of Qom country in Iran 
Figure 3 is the image of Kuh Sefid district acquired from Google Earth.

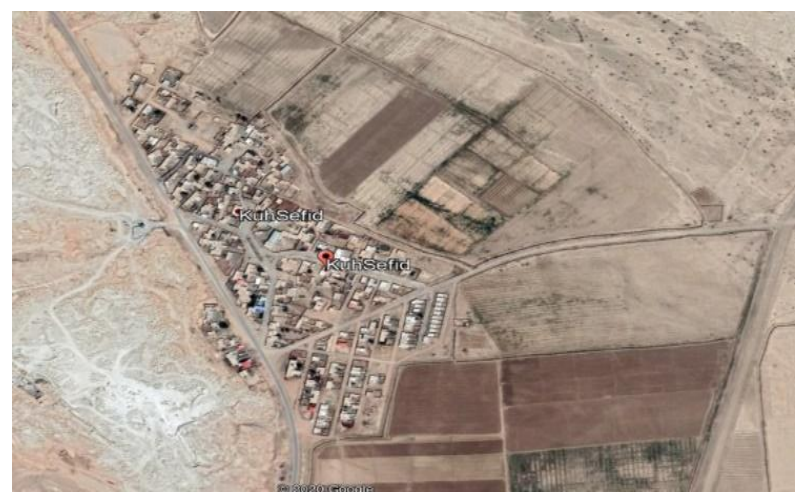

Figure 3. Google Earth image

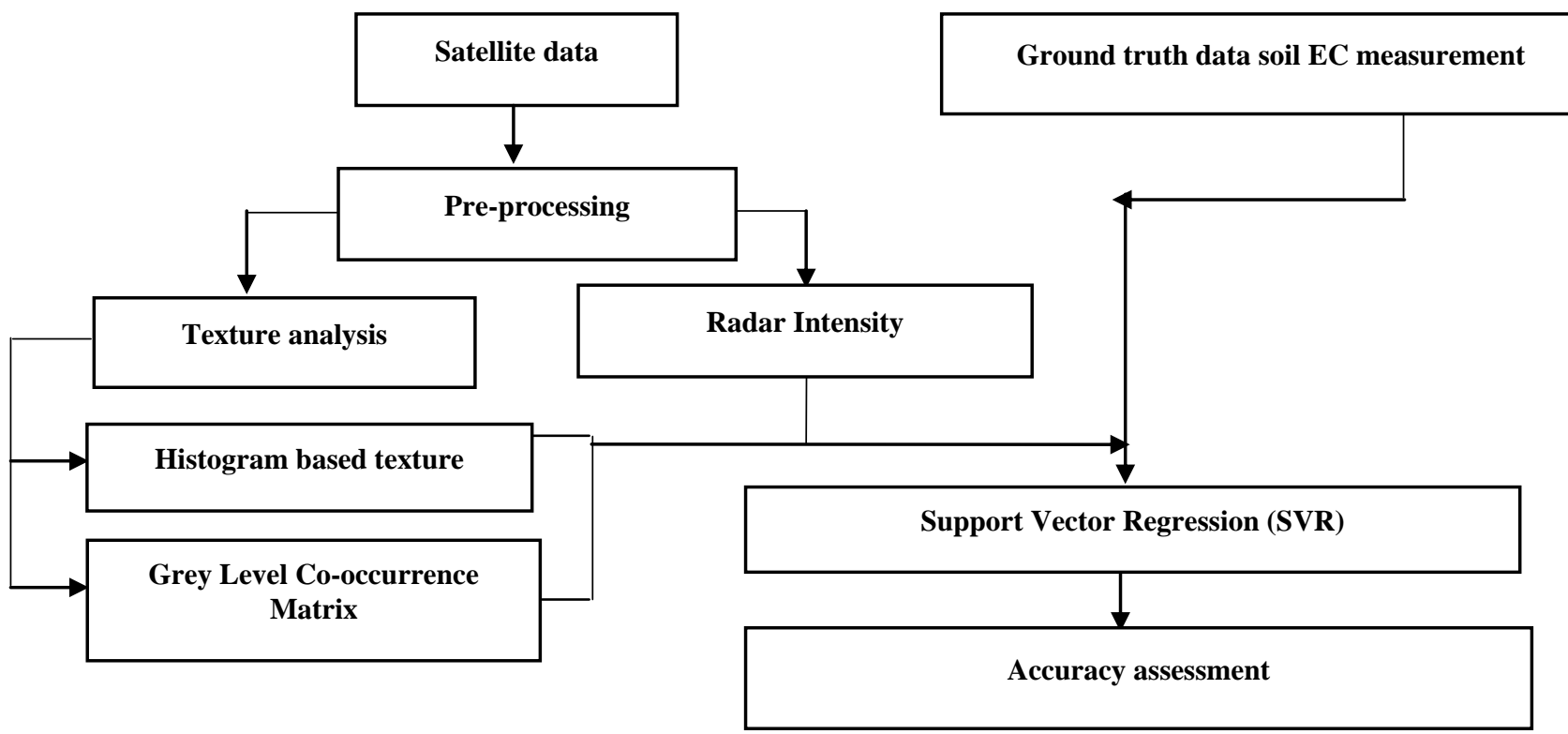

Figure 4. Flowchart of Method

\section{PERFORMANCE EVALUATION METRICS}

The parameters with the help of which accuracy assessment is done is given below.

\subsection{Root Mean Square Error (RMSE)}

Mean square error helps to find the error between predicted value and actual value over samples. If $\widehat{y}_{i}$ is the predicted value, and $y_{i}$ is the corresponding true values, then the mean squared error (MSE) estimated over $n_{\text {samples }}$ is defined as:

$\operatorname{MSE}(y, \hat{y})=\frac{1}{n_{\text {samples }}} \sum_{i=0}^{n_{\text {samples }}-1}\left(y_{i}-\widehat{\left.y_{i}\right)^{2}}\right.$

Where, root mean square error is the square root of mean square error.

\subsection{Normalized Root Mean Square Error (NRMSE)}

The Normalized root mean square error is expressed as :

$N R M S E=\frac{R M S E}{\bar{y}}$

\subsection{Coefficient Of Determination $\left(\mathbf{R}^{\mathbf{2}}\right)$}

The $r 2$ score function, for the most part denoted as $R^{2}$. It determines how well the samples are estimated by the model.

\subsection{Field Investigation}

The field observations were done on $4^{\text {th }}$ March 2017, and 58 soil tests were randomly gathered.

\subsection{Used Methodology}

The following section depicts the method employed to meet the goal of this work. The outline of the method adopted for this work has been shown in figure (4). In this figure, the approach is to estimate soil electrical conductivity using support vector regression with $\mathrm{K}$ - Fold technique. In this project, approach is to estimate soil electrical conductivity from the Sentinel -1 SAR image[9]. So the satellite dataset was available in SLC format, therefore pre - processing was carried out. Then radar intensity and texture features were derived[8]. The SVR algorithm with K-Fold technique was applied with radial basis function as a kernel.

\section{Ground truth data soil EC measurement}

If $\widehat{y}_{i}$ is the predicted value and $y_{i}$ is the corresponding true value for total $\mathrm{n}$ samples, the estimated $\mathrm{R}^{2}$ is:

$$
R^{2}(y, \hat{y})=1-\frac{\sum_{i=1}^{n}\left(y_{i}-\widehat{y_{i}}\right)^{2}}{\sum_{i=1}^{n}\left(y_{i}-\bar{y}\right)^{2}}
$$

\section{RESULTS AND DISCUSSIONS}

This section discusses the performance of algorithm on the basis of quantitative assessment values. In this, radial basis function (RBF) is used as a kernel function.

Table 1. Accuracy Assessment

\begin{tabular}{|c|c|c|c|}
\hline K-Fold & RMSE & NRMSE & $\mathbf{R}^{\mathbf{2}}$ \\
\hline 5 & 15.33 & 1.209 & 0.059 \\
\hline 10 & 11.51 & 0.908 & 0.229 \\
\hline 15 & 4.06 & 0.32 & 0.593 \\
\hline 29 & 0.146 & 0.011 & 0.998 \\
\hline 35 & 0.014 & 0.0011 & 0.0 \\
\hline 40 & 0.014 & 0.0011 & 0.0 \\
\hline 45 & 0.014 & 0.0011 & 0.0 \\
\hline 58 & 0.014 & 0.0011 & 0.0 \\
\hline
\end{tabular}


As it can be seen from table 1, the better model is achieved when values of $\mathrm{K}$ is 29.Accordingly, RMSE value is 0.146 and $\mathrm{R}^{2}$ value is 0.998 .

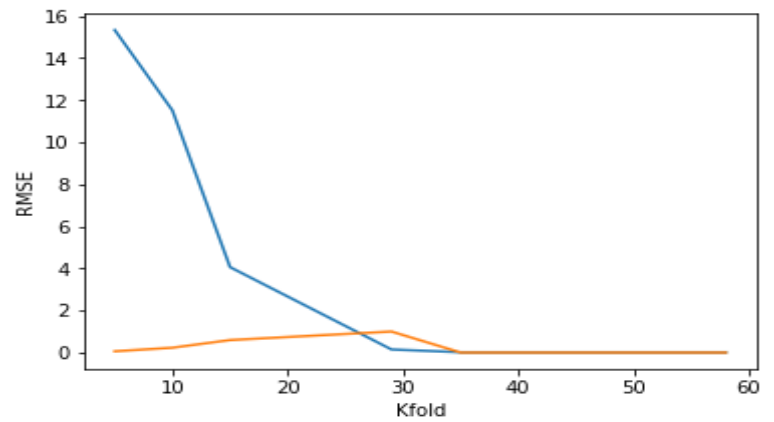

Figure 5.Graph showing variation of RMSE and $\mathbf{R}^{\mathbf{2}}$ w.r.t.K value

The graph plotted showing the variation of K- Fold with RMSE and $R^{2}$ values is given in figure 5. The blue line indicates the variation of $\mathrm{R}^{2}$ with $\mathrm{K}$ - Fold and orange line indicates the variation of RMSE with K-Fold. As it can be seen from graph, when value of $\mathrm{k}$ is $29, \mathrm{RMSE}$ is minimum and $\mathrm{R}^{2}$ is 0.998 .

\section{CONCLUSION}

This paper has concentrated on assessing the capability of Sentinel -1 data in checking soil saltiness. This study helps to determine soil salinity with the help of Sentinel -1 data and then assessing the performance of algorithm on the basis of performance evaluation metrics. From the observations, it can be concluded that, for value of $\mathrm{K}$ as 29 , we are getting root mean square error as 0.146 and $\mathrm{R}^{2}$ as 0.998

\section{REFERENCES}

[1] Mohammad Mahdi Taghadosi, Mahdi Hasanlou, Kamran Eftekhari, 2019, "Soil salinity mapping using dual polarized SAR sentinel -1 imagery", International Journal of Remote Sensing.

[2] Ashutosh Pandey, Shashi Ranjan Kumar, A.K. Shrivastava, 2018, "Review and analysis of Microwave
Remote Sensing behavior of soil in India", International Journal of Research in Advent Technology, vol. 6, No. 1 .

[3] Agnieszka Szyplowska, Justyna Szerement, Arkadiusz Lewandowski, 2018, "Impact of soil salinity on the relation between soil moisture and dielectric permittivity", IEEE.

[4] Hong Jiang, Yusufujiang Rusuli, Tureniguli Amuti \& Qing He, 2018, "Quantitative assessment of soil salinity using multi - source remote sensing data based on the support vector machine and artificial neural network", International Journal Of Remote Sensing.

[5] Singh, G., D.S. Bundela, M. Sethi, K. Lal and S.K. Kamra, 2010,"Remote Sensing and geographic information system for appraisal of salt - affected soils in India", Journal Of Environmental Quality.

[6] Saha, S.K., 2011, "Microwave Remote Sensing in Soil Quality Assessment", ISPRS - International Archives of the Photogrammetry, remote sensing and spatial information Sciences.

[7] [Wu, Y., and W.Wang, 2011, "Modelling the backscattering coefficient of salt affected soils using AIEM model", In Earth Resources and environmental remote sensing/GIS application II, International society of optics and photonics.

[8] Li,Y.Y., K.Zhao, J.H. Ren, Y.L. Ding, and L.L. Wu.,2014, "Analysis of dielectric constant of saline alkali soils and the effect on Radar backscattering coefficient: A case study of soda alkaline saline soils in western Jilin province using RADARSAT-2 data", The Scientific World Journal.

[9] Vijayarekha, K., 2014, "Feature Extraction", School of Electrical and Electronics Engineering.

[10] Xiangzheng Meng, Qi Zhou, Jiexiang $\mathrm{Hu}$, Leshi Shu,Ping Jiang, 2017, "A Global Support Vector Regression based on sorted K - Fold Method", IEEE International Conference on Industrial Engineering and Engineering Management. 